\title{
BIBECHANA
}

A Multidisciplinary Journal of Science, Technology and Mathematics

ISSN 2091-0762 (online)

Journal homepage: http://nepjol.info/index.php/BIBECHANA

\section{A Brief review on extraction of nanocellulose and its application}

\author{
Jyoti Giri ${ }^{1}$, Rameshwar Adhikari ${ }^{2, *}$ \\ ${ }^{1}$ Department of Chemistry, Tri-Chandra Campus, Tribhuvan University, Kathmandu, Nepal \\ ${ }^{2}$ Central Department of Chemistry, Tribhuvan University, Kirtipur, Kathmandu, Nepal \\ * Corresponding author: E mail: nepalpolymer@yahoo.com \\ Article history: Received 22 October, 2012; Accepted 7 November, 2012
}

\begin{abstract}
Cellulose is one of the most abundant renewable natural resources. With the advent of nanotechnology, the interests of cellulose scientists have diverted towards the extraction of nanocellulose from various plant sources and utilize them in technical applications. The reason behind the rapid progress in the nanocellulose chemistry and engineering lies in the promising properties of the nanocellulose and the products thereof. In fact, nanocelluloses combine the important properties of cellulose with amazing features of nanoscale materials. With the perspective of potential of cellulosic materials production in Nepal basically from agricultural wastes and uses in several applications such as in the preparation of biomaterials, this paper reviews the current knowledge in research and development of nanocelluloses in particular for biomedical applications. After the introduction to the chemical constitution and microfibrillar arrangement of nanocellulose in the cellulose bundles together with other constituents, the ways of preparation of nanocellulose and its functionalization approaches will be discussed. Finally, the application of nanocellulose for preparing biomaterials scaffolds will be introduced
\end{abstract}

Keywords: Nanocellulose; tissue engineering; functionalization; biopolymer

\section{Introduction}

Cellulose is main lignocellulosic component of cell wall in plants along with hemicellulose, lignin, pectin, wax and constitutes $25-50 \%$ of the plants [1]. Cellulose can be replenished by photosynthesis and its estimated biosynthesis is $10^{11}$ tons per year [2]. The simple molecular structure of cellulose is given in Fig. 1.

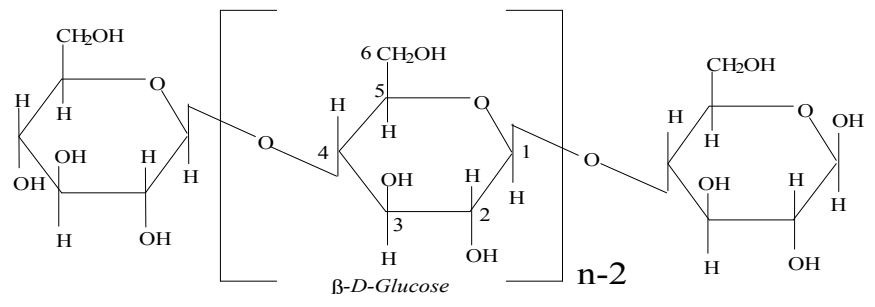

Fig. 1: Structure of cellulose macromolecule. 
Cellulose is linear polymer of $\beta$-anhydroglucopyranoside with $1,4 \beta$-glycosidic linkage. The structure is supported by the free secondary $\mathrm{OH}$ groups at $\mathrm{C}-2, \mathrm{C}-3$ position and primary $\mathrm{OH}$ group at C-6 position $[1,3,4]$. Its general molecular formula is $\left(\mathrm{C}_{6} \mathrm{H}_{10} \mathrm{O}_{5}\right)_{\mathrm{n}}$ and density is $1.50 \mathrm{~g} / \mathrm{cm}^{3}$. About several hundred to10 millions of glucose units condense to form a straight chain of a polysaccharide unit in the term of cellulose nanofiber. The free $\mathrm{OH}$ groups in one polysaccharide thread have higher possibilities to form hydrogen bonds with another thread. Therefore a number of nanofibers bind through intermolecular hydrogen-bonding with each other to form microfibers and then to microscopic cellulose fibers. The cellulose macromolecule assembled in semicrystalline filament is expressed in term of microfibers and nanofibers. The $\mathrm{OH}$ groups are also the active sites for the chemical modifications [5].

Hemicellulose is amorphous polysaccharide which is a mixture of carbohydrates comprising 3- 6 membered units [1,6]. Lignin is complex dendridic network of phenyl propene which acts as binder in cellulose fiber to give the exact morphology for plant cell wall [1,6]. Similarly pectin is heteropolysaccharide of 1-4 linked galacturonic acid with methyl esters of different sugar units [1,6]. Wax has various composition of esters obtained from fatty acids and long chain alcohols. e.g. rice bran wax, soy wax etc. $[2,6]$.

Cellulose occurs in 4 major polymorphic forms: cellulose I, II, III and IV. Cellulose fibrils are either manmade (regenerated also called fibers) or natural. Cellulose with these two origins has predominantly structures I and $\mathrm{II}^{2}$. In cellulose I crystal structure has parallel unit cell whereas in cellulose II unit cells of crystals are antiparallel ${ }^{2}$. Cellulose III is obtained on treating cellulose I and II with ammonia. Likewise cellulose IV can be prepared by heating cellulose III [7]. Although native Cellulose has I structure, it can be converted into other forms by different treatments. Cellulose fibrils are partly crystalline with cellulose $\mathrm{I}_{\alpha}$ and $\mathrm{I}_{\beta}$. Cellulose $\mathrm{I}_{\alpha}$ has a single chain triclinic structure and $\mathrm{I}_{\beta}$ has two chain monoclinic structures $^{2}$. These special arrangements provide the cellulose nanofibers with remarkably interesting mechanical properties [8].

Lignin, hemicellulose and wax matrix bind the cellulose fibers in plant cell wall. In a single fiber, a number of threads like microfibrils are bound together which are called microfibers. Their size varies from 10 to $40 \mu \mathrm{m}$ in diameter depending upon the types of plants; see Table 1 [9].

Table 1: Average fiber dimensions of some cellulose raw materials [9].

\begin{tabular}{lcc}
\hline Cellulose material & Fiber length $(\boldsymbol{\mu m})$ & Fiber width $(\boldsymbol{\mu m})$ \\
\hline Spruce & 3400 & 31 \\
Pine & 3100 & 25 \\
Beech & 1200 & 21 \\
Eucalypt & 850 & 20 \\
Bamboo & 2700 & 14 \\
Cotton linters & 9000 & 19 \\
Wheat straw & 1410 & 15 \\
Baggage & 1700 & 20 \\
\hline
\end{tabular}

Each microfiber is a bundle of individual nanosize thread, called as nanofiber or nanofibril which consists of a number of filamental cellulose chain passing through numerous crystallites in thread like fashion. The elementary nanofibril has diameter of 3-15 nm and length with an average aspect ratio of 20-200 [10]. These nanofibrils contain ordered nanocrystallites and disordered amorphous domains placed in definite intervals [11].

Cellulose fibers being most abundant, constantly replenished in nature by photosynthesis [2,12] and being stronger than steel, glass fiber etc, has attracted new trends of development in material science [13,14]. 
High mechanical strength, stiffness, large surface area and biodegradability are the properties often referred to nanocellulose which make it as tempting raw material for new biobased composites. Around 1990, their strong hydrophilic nature, resistant to many chemicals, safety to life, reproducibility and recyclability, brought nanocellulose in many intense applications compared to cellulose and microcellulose [15]. Additionally the recent advances in nanocellulose supports medical fields in different aspect as implant materials (artificial organs), biodegradable tissue scaffolds, drug delivery vehicles, etc. Further nanofiber composites are used for making flexible circuits, solar panels and other electronics devices. Many more applications in paints, pigment, inks, screens, coatings, packaging materials, optical appliances, cosmetics etc. also make use of cellulosic materials $[15,16]$.

The aim of this paper is to review the extraction methods of nanocellulose from renewable natural resources and give an overview of various applications as novel biomaterials.

\section{Extraction Micro- and Nanocellulose}

With the beginning of civilization cellulose is being used for many purpose; such as for making clothes, ropes, housing materials and paper from fiber. Time passed by and new ideas with novel developments started. In late 1970s, Turbak, Snyder and Samberg introduced the new material microfibrillated (MFC) and nanofibrillated cellulose (NFC) [17]. Cotton [11,12], ramie [11], jute [11,18], bamboo [19,22], sisal [9], rice [19], starch [21] etc have been used to extract nanocellulose.

There are various procedures for the preparation of micro and nanofibrillated cellulose. Deshpande \& coworkers had extracted microfibrillated cellulose by compression and roller mechanical techniques and blended with polyesters to prepare high strength composite materials [19]. Rajan \& co-workers microfibrillated bamboo fibers by chemical treatment with $\mathrm{NaOCl} / \mathrm{NaOH}$ and acetic acid in an autoclave which were then compounded with polyhydroxybutyrate (PHB) matrix [22]. Similarly, Okubo, Fujii \& Yamashita extracted microfibers by steam explosion process and developed composites with polylactic acid (PLA) matrix, whose bending, fracture properties were improved [18].

In nature even the waste materials could be of high value as they could be one of the sources for raw material to isolate micro-nanocellulose. Sun \& co-workes and Mandal \& co-workers isolated cellulose microfibers from the waste sugarcane dewaxed bagasse by chemical treatments followed by ultasonication process and oxidation with hydrogen peroxide $\left(\mathrm{H}_{2} \mathrm{O}_{2}\right)$ and sodium hydroxide $(\mathrm{NaOH})$ $[2,12]$.

Liu, Yuan \& Bhattacharyya extracted suspension of nanocellulose fiber from flax yarns. Mercerized flax yarns on acid treatment and neutralization with alkali yielded nanocellulose. The PLA composites with these nanofibers showed significant increase in tensile strength and modulus whereas elongation at break was decreased with fiber content [23]. Cherian \& co-workers used pineapple leaf fiber (PALF) to extract nanocellulose by chemical as well as mechanical process. Fibers on delignification, bleaching and steam explosion gives nanocellulose width in the range of 5-60 $\mathrm{nm}$ [24]. Kukle \& co-workers optimized the percentage of $\mathrm{NaOH}$, temperature, time and pressure for nanoscaled disintegration of hemp fibers after steam explosion to isolate the nanocellulose [8]. Likewise, Luduena and co-workers obtained nanocellulose from rice husk by chemical process [25].

In the survey it is found that there are many different methods for the extraction / preparations of cellulose, cellulose micro and nanofibrils which can be summarized as chemical and mechanical processes [13].

\section{Chemical process}

The chemical process comprises the treatment of raw cellulosic mass with required amount of alkali for delignification, organosolvation (with acetic acid, aqueous methanol or ethanol), and acid treatment 
for the hydrolysis. Chemical process also involves oxidation with oxidizing agent hydrogen peroxide $\left(\mathrm{H}_{2} \mathrm{O}_{2}\right)$, sodium hypochlorite $(\mathrm{NaOCl})$ for bleaching the coloured materials.
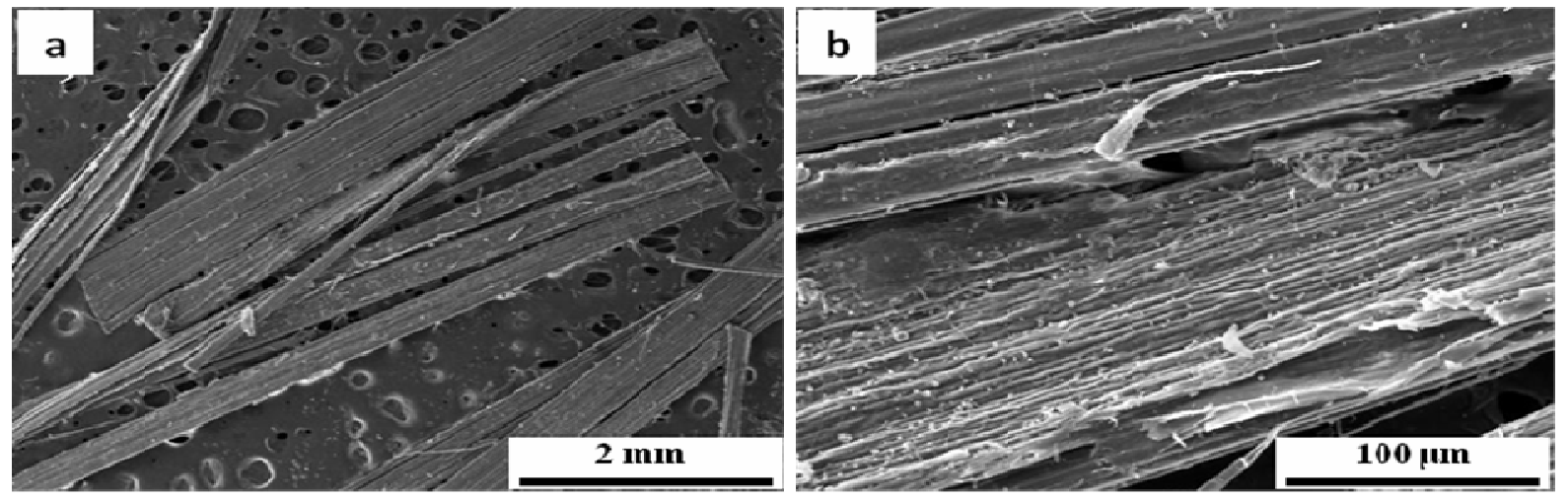

Fig. 2: SEM images of chemical treated hemp fiber: [a] Washed, [ b] delignified [10].

\section{Mechanical process}

Mechanical process can be carried out in different ways: roller mechanical technique, compression mechanical technique, cryocrushing, sonication, homogenization and wet cooking process (steam explosion).

\subsection{Compression (CMT) and roller mechanical (RMT) techniques}

In CMT delignified fibers of cellulosic materials are placed in a bed of stripes placed between the two plates and subjected to a constant load of 10 tons for 10 seconds. In contrast, RMT delignified stripes are forced between the two rollers, one of which is fixed while the other is rotating [3].
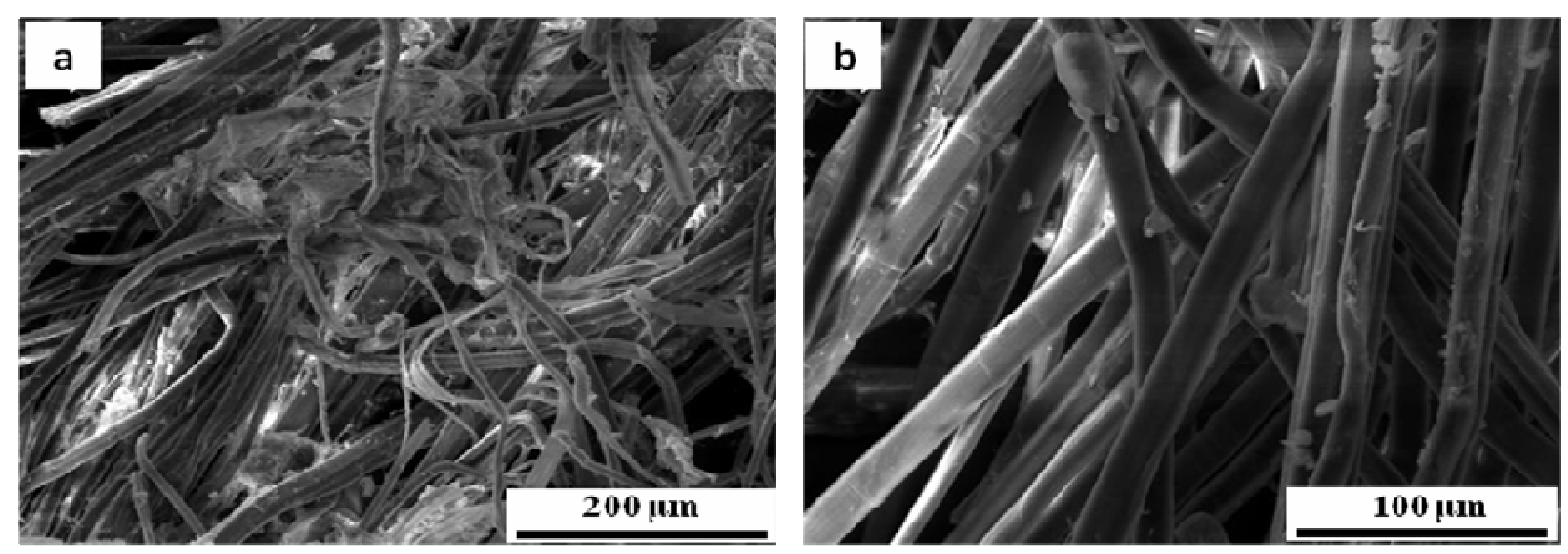

Fig. 3: SEM images of mechanical and chemical treated delignified hemp fiber for micro, nanofibrillation: [a] steam exploded, [b] acid hydrolysed steam exploded fibers [8].

\subsection{Homogenization}

In this process fibers (either crude one or delignified ones) are passed through a valve at high pressure and exposed to a pressure drop to atmospheric condition when the valve is released resulting in high shear force on the fiber surface [3,21]. 


\subsection{Cryocrushing}

The fibers are first frozen in liquid nitrogen. The embrittled glassy fibers are then subjected to high speed crushing. The high shear and impact forces acting on the fibers turn them to powder comprising microfibrils. The cryocrushed fibers may then be dispersed uniformly into water suspension using a disintegrator [21].

\subsection{Steam explosion}

It is a thermomechanical process to breakdown the structural components of cellulose. The process is accompanied with heat carried by steam. Steam at high pressure penetrates the lignocellulosic biomass through diffusion. The sudden release of pressure generates shear force which hydrolyze the glycosidic bond and hydrogen bonds between the glucose chains, leading to the formation of nanofibers [24].

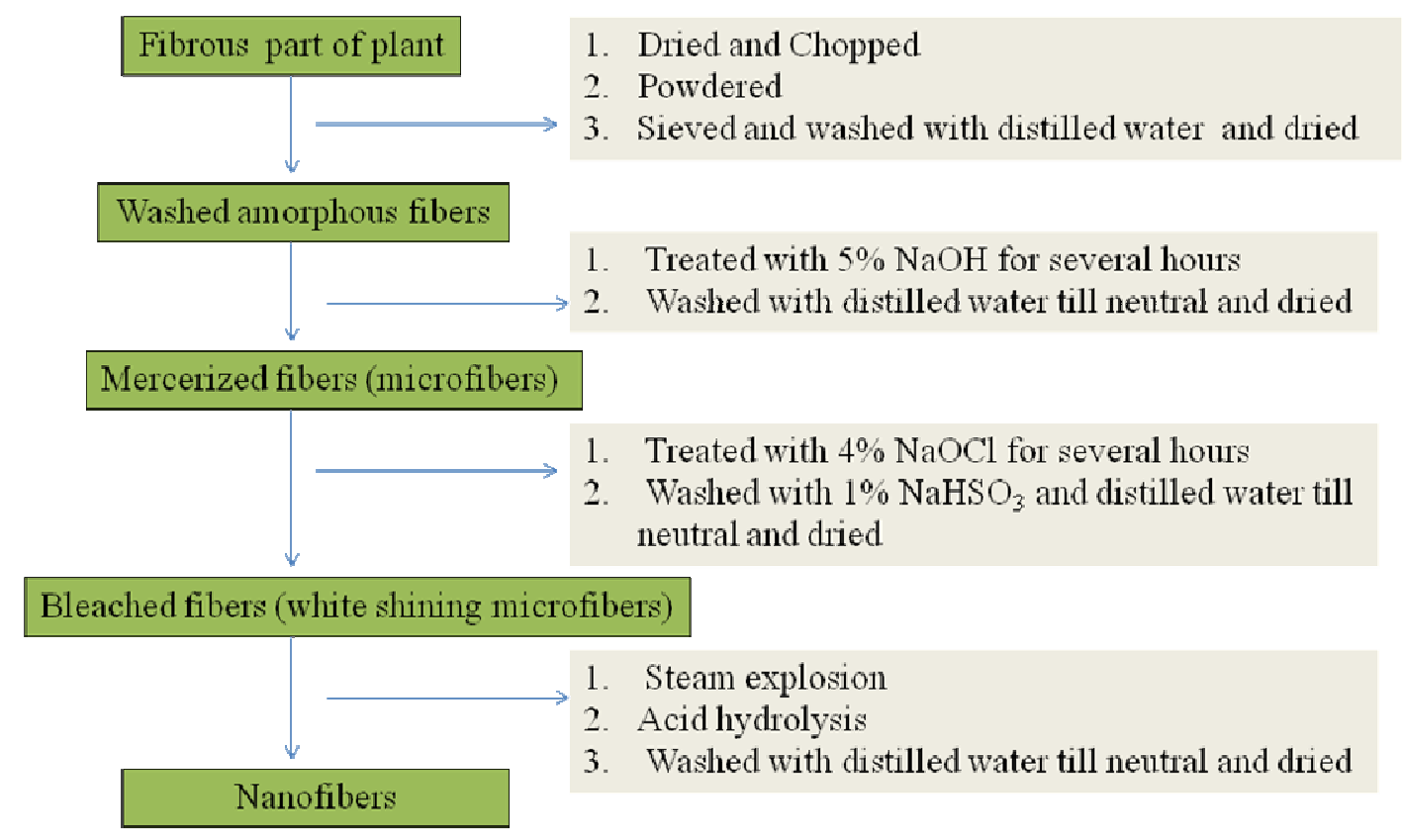

\section{Fig. 4: Scheme for nanofibers extraction from lignocellulosic materials using chemical and mechanical treatments.}

It is convenient method and common practice both chemical and mechanical processes to prepare nanocellulose from lignocellulosic materials. The unified chemical/mechanical scheme is presented in Fig. 4.

\section{Applications}

The nanofibers obtained have several potential applications in the field of electronics, used to prepare flexible circuits, flexible solar panels, optical applications, etc [5]. For instance, the acetate functional derivatives of sisal cellulose have been found to be applicable in textile (clothing and fabric), high absorbency products (diapers, cigarette- filters and other filters), thermoplastic products (films and plastic instruments), nourishing (food packaging), cosmetics and pharmaceuticals (extended capsule, tablet release agents and encapsulating agents), medicinal (hypoallergenic surgical products) and others [16]. 
Cellulose nanocrystals with defined dimension formulate the cellulose based nano composites for different coating applications and in packaging materials. Cellulose bears high capacity to hold water, and therefore has higher compatibility with human body. Thus nanocellulose can be used to fabricate tissue scaffolds and implants, wound dressing, biocompatible coatings and drug release formulations.

\section{Concluding Remarks}

Nanocellulose is an interesting material with amazing properties and can be applied for many useful purposes such as in textile industries, nanomedicine, food packaging, cosmetics and pharmacy. Other potential applications are use as polymer nanocomposites in combination with other polymers, hydrogels and technical materials. The research works envisioned in our laboratory are focusing on the extraction, chemical modification and applications of nanocellulose especially in the preparation of composite materials, biomedical and tissue engineering devices with the aid of electrospinning. In the frame of a University Grants Commission (UGC) supported project, we are working to develop standard protocols to extract the nanocellulose from some agricultural wastes and prepare useful devices for biomedical applications.

\section{Acknowledgements}

The authors would like to thank the University Grants Commission (UGC), Nepal for providing us with the Faculty Research Grant 2068/2069 (Project: Extraction of Micro- and Nanofibers from Renewable Resources to Develop Completely Biodegradable Plastics Composites).

\section{References}

[1] T.T. Teeri , H. Brumer-III, G. Daniel and P. Gatenholm Trends in Biotechnology, 25 (2007) 299-306.

[2] J.X. Sun, X.F. Sun, H. Zhao and R.C. Sun, Polymer Degradation Stability, 84 (2004) 331-339.

[3] Stelte W. and Sanadi A.R., Preparation and Characterization of Cellulose Nanofibers from Two Commercial Hardwood and Softwood Pulps, Industrial Engineering and Chemical Research, 48 (2009) 11211-11219.

[4] J.Yang and D.Y. Ye, Chinese Chemical Letters, 23 (2012) 367-370.

[5] D. Klemm, B. Philipp, T. Heinze, U. Heinze and W. Wagenknecht, Comprehensive Cellulose Chemistry, Functionalization of Cellulose, Volume II, WILEY-VCH Verlag GmbH,Weinheim (1998).

[6] S.J. Eichhorn, C.A. Baillie, N. Zafeiropoulos, L.Y. Mwailambo, M.P. Ansell, A. Dufresne, K.M. Entwistle, P.J. Herrere-Franco, G.C. Escamilla, L. Groom, M. Hughes, C. Hill, T.G. Rials and P.M. Wild, Journal of Materials Science, 36 (2001) 2107-2131.

[7] S. Park, J.O. Baker, M. E. Himmel, P.A. Parilla and D. K. Johnson, Biotechnology for Biofuels, 3(2010)10.

[8] S. Kukle, J. Gravitis, A. Putnina and A. Stikute. Proceedings of $8^{\text {th }}$ International Scientific and Practical Conference., 1 (2011) 230-237.

[9] D. Klemm, B. Philipp, T. Heinze, U. Heinze and W. Wagenknecht, Comprehensive Cellulose Chemistry, Fundamentals and Analytical Methods, Volume I, WILEY-VCH Verlag GmbH,Weinheim (1998).

[10] W.J. Orts, J. Shey, S.H. Imam, G.M. Glenn, M.E. Guttman and J.F. Revol, Journal of Polymers and Environment, 13 (2005) 301-306.

[11] M. Ioelovich, BioResources, 3 (2008) 1403-1418.

[12] A. Mandal and D. Chakrabarty, Carbohydrate Polymers, 86 (2011) 1291-1299.

[13] F.Y. Yan, D. Krishniah, M. Rajin, and A. Bono, Journal of Engineering Science and Technology, 4 (2009) 57-68.

[14] D.M. Panaitescu, A.N. Frone, M. Ghiurea, C.I. Spataru, C. Radovici and M. D. Iorga, Properties of Polymer Composites with Cellulose Microfibrils, In: Advances in Composite Materials- Ecodesign and Analysis, Brahim Attaf (Ed.), InTake Open Access Book (2011).

[15] Y.H. Han, S.O. Han, D. Cho and H. Kim-II, Macromolecular Research, 16 (2008) 253-260.

[16] M.P.D. Paula, T.M. Lacerda and E. Frollini, eXPRESS Polymer Letters, 2 (2008) 423-428. 
[17] A.F. Turbak, F.W. Snyder and K.R. Sandberg, Journal of applied Polymer Science, Applied Polymer Symposia, 37 (1983) 815-827.

[18] K. Okubo, T. Fujii and N. Yamashita, JSME International Journal, 48 (2005) 199-204.

[19] A.P. Deshpande, M.B. Rao and C. L. Rao, Journal of Applied Polymer Science., 76 (2000) 83-92.

[20] K.P. Rajan, N.R. Veena, H.J. Maria, R. Rajan, M. Skrifvars and K. Joseph, Journal of Polymers and Environment, 17 (2009) 109-114.

[21] I. Siro and D. Plackett, Cellulose., 17 (2010) 459-494.

[22] K.P. Rajan, N.R. Veena, H.J. Maria, R. Rajan, Journal of Composite Materials, 45 (2010) 1325-1329.

[23] D.Y. Liu, X.W. Yuan, D. Bhattacharyya and A.J. Easteal, eXPRESS Polymer Letters, 4 (2010) 26-31.

[24] B.M. Cherian, A.L. Leao, S.F. De-Souza, S. Thomas, L.A. Pothan and M. Kottaisamy, Carbohydrate Polymers, 81 (2010) 720-725.

[25] L. Luduena, D. Fasce, V.A. Alvarez and P. M. Stefani, BioResoources, 6 (2011) 1440-1453. 